\title{
The Association between Pre- and Post-Migration Wellbeing Factors with Integration among Russian, Somali, and Kurdish Origin Adults in Finland
}

\author{
ELFADL W, Uppsala University \\ International Maternal and Child Health, Department of Women's and Children's Health, Sweden \\ LILJA E, Finnish Institute for Health and Welfare \\ Equality and Inclusion Unit, Finland
}

SKOGBERG N, Finnish Institute for Health and Welfare

Equality and Inclusion Unit, Finland

SELLING K, Uppsala University

International Maternal and Child Health, Department of Women's and Children's Health, Sweden

CASTANEDA AE, Finnish Institute for Health and Welfare

Equality and Inclusion Unit

Corresponding author:

DR ANU E CASTANEDA, Finnish Institute for Health and Welfare Helsinki, Finland

\section{Abstract}

Pre- and post-migration related factors are likely to influence integration outcomes of migrants. This study aimed to investigate which pre-migration factors (basic education and potentially traumatic experiences) and post-migration wellbeing factors (quality of life and loneliness) are associated with integration outcomes (employment status, language skills, voting, media use, having host country's native friends, participation in leisure activities) of migrant background men and women. The Finnish Migrant Health and Wellbeing Study (Maamu) was used, including working-aged adults of Russian, Somali, and Kurdish origin. Each integration outcome was analysed by each predictive factor with logistic regression, separately for the three groups and separately for men and women. The integration outcomes were somewhat differently associated with pre- and post-migration factors in the different groups. All these aspects are important to be taken into account in integration discourse to promote both integration and social wellbeing.

Keywords: integration, migrant, migration, wellbeing, population-based study, Maamu 


\section{Introduction}

The global migration trend has changed over the recent decades regarding origins and destinations as well as the volume and the type of migration (Skeldon 2013). Globally more than $70 \%$ of migrant population are of working age and half are women (United Nations 2016). The increase of the number of migrants in Finland, too, has been rapid during the recent decades (Statistics Finland 2020).

One of the key concepts in migration discourse is integration. It is a multidimensional concept and there are numerous ways in how it is defined. According to one of the most known integration theoretics, J.W. Berry (1997, 2005), integration happens when the cultural integrity and ethnic identity is maintained while participating as an active member in daily life in the new larger society. Integration can be achieved when both minor and major groups are tolerant to new cultures. The European Union defines integration as a bidirectional process where the newcomers and the local community have both active roles (Martiniello 2006). The Finnish integration policy defines integration in a similar manner: as a personal process of migrants for participation in working life and society while maintaining their own language and culture (Ministry of Employment and the Economy 2010). The Act of the Promotion of Immigrants Integration in Finland has been instilled in order to support and promote integration and enable migrants to be active members of the society.

There are several ways of operationalizing the phenomena of integration in quantitative research, both from the minor and major groups' perspectives. Some of the generally used factors for minority groups' integration include working, studying or having other similar activities to participate in the society, understanding and speaking the languages of the new host country, having a sense of belongingness, having friends and other social networks in the new host country, having hobbies or other ways to participate in the daily leisure time activities, following local media, voting in local elections, perceiving the life in the new host country meaningful and enjoyable, and so on. Correspondingly, the major groups' perspectives would include for example attitudes.

Some challenges in migrants' integration have been observed in Finland. For example the Finnish Migrant Health and Wellbeing Study (Maamu) that investigated the health and wellbeing of the Russian, Somali, and Kurdish origin adults showed for instance that unemployment rates were high, only less than one third of the Kurdish origin migrants reported having a good Finnish friend, and 29 percent of the Somali origin women reported poor command of Finnish or Swedish language, even though the mean time living in Finland was from 11 to 12 years (Castaneda et al. 2012).

Since integration is a priority on a policy level and has immense human capital advantages, factors affecting integration outcomes need to be identified, with respect to both protective and challenging perspectives. In the Finnish context, a recent study showed that having social networks associates positively with good command in Finnish or Swedish, being employed, voting, and having high sense of belongingness (Castaneda and Kuusio 2019). Perceived discrimination in the new host country has also been shown to have an association with decreased trust towards society and feeling unsafe (Castaneda 
et al. 2015). However, despite the well-known importance of integration, the evidence of the factors affecting it is still scarce.

There is also a debate whether integration is merely challenged by pre-migration factors, such as lack of education, traumatic experiences or other problems in the former home country, or post-migration factors, such as perceived discrimination or other wellbeing factors in the new society. This is an important question, since it would be important to know where to allocate the resources when trying to promote integration. For instance, people coming from conflict areas are known to have high prevalence of potentially traumatic experiences (Castaneda et al. 2017), but the knowledge of the linkup between traumatization and integration is still lacking.

Even though both men and women might have vulnerabilities as migrants, women are often thought of having even a more vulnerable position as being the historically suppressed party. Many health and wellbeing disadvantages are seen among the migrant origin women worldwide and in Finland, too. Migrant origin women tend to have for instance more mental health problems (Rask et al. 2016a) and poorer physical health, including a higher prevalence of chronic health problems (Skogberg and Koponen 2019), obesity (Skogberg et al. 2018), and functional capacity limitations (Rask et al. 2016b) than their male counterparts or than women in the general population. On the other hand, migrant origin men have for instance more substance use (Salama et al. 2018) and experiences of torture (Castaneda et al. 2017) than migrant origin women. Therefore, gender-specific aspects are important to be taken into account.

This study aimed to investigate the associations between pre-migration (potentially traumatic experiences and educational level) and post-migration (quality of life and loneliness) wellbeing factors with integration outcomes (employment status, host country's language skills, voting in elections, local media use, having friends with Finnish background, and active participation in leisure activities) among Russian, Somali, and Kurdish origin men and women in Finland. It was hypothesized that protective pre- and post-migration situations and social wellbeing factors, here higher educational level, having no potentially traumatic experiences, having higher quality of life, and not having feelings of loneliness, associate with better integration outcomes, here being more often employed, having good command in Finnish or Swedish, voting more often, using local media more often, having friends with Finnish background, and participating actively in leisure activities.

\section{Methods}

\section{Study setting}

The data was drawn from the Finnish Migrant Health and Wellbeing Study (Maamu), which was conducted in Finland 2010-2012 by the Finnish Institute for Health and Welfare (THL). Three migrant origin groups were included in the study, Russian, Somali, and Kurdish origin adults, with a study sample of 1,000 persons in each group living in six big Finnish cities (Helsinki, Espoo, Vantaa, Turku, Tampere, and Vaasa), randomly selected 
from the National Population Register. The sampled persons were aged between 18 to 64 years and had lived in Finland for at least one year.

The inclusion criteria for the Russian origin group were defined as persons born in Russia or former Soviet Union and having Russian or Finnish as their mother tongue. Somali origin group was defined as individuals born in Somalia. For Kurdish origin group it was required to be born in Iraq or Iran and having Kurdish as mother tongue. Persons still living in reception centres did not fulfil the study inclusion criteria.

The Maamu study included a structured face-to-face interview lasting approx. 1-1.5 hours and a standardized health examination lasting approx. one hour. A short version of the interview was offered to those who did not want to participate in the longer version. The interview included a broad range of topics on health and wellbeing. The interviews and health examinations were carried out by a team that spoke both Finnish and the language of the target groups. A total of $70 \%(n=702)$ for the Russian origin group, $51 \%$ $(n=512)$ for the Somali origin group, and 63\% $(n=632)$ for the Kurdish origin group participated in at least one part of the study. Participation rate was higher among women, Russian, non-metropolitan area residents, married, and with higher age. More detailed description of the study is provided elsewhere (Castaneda et al. 2018).

\section{Predictors}

Two pre-migration wellbeing factors were used: the level of basic education and potentially traumatic experiences in the former home country.

The level of basic education was assessed in both the full and short interviews by asking "What is your basic education?" with answer options "no education", "primary school or part of it", "secondary school or part of it", "high school or part of it", and "other basic education", dichotomised into "high school" (high school or part of it) versus "comprehensive school or lower" (no education, primary school, or secondary school).

Potentially traumatic experiences in the former home country were investigated in the full interview by eight sub-questions: "Have you been involved in war? Experienced natural disaster? Seen a violent death or injury? Faced sexual violence? Experienced physical attack? Been captured or kidnapped? Being tortured? Experienced other type of serious violence?", each answered by "yes" or "no". The questions were combined and dichotomised into "having potentially traumatic experiences" (at least one of the eight answered yes) versus "not having traumatic experiences" (all of the eight answered no).

Two post-migration wellbeing factors were used: quality of life and feelings of loneliness. These factors were used as predictors in the present study's statistical models, but can be interpreted also as outcomes since truly only the associations between factors are investigated.

Quality of life was assessed in the full interview by asking "How do you evaluate your quality of life?" with answer options "very good", "good", "not good nor bad", "bad", and "very bad", dichotomised into "good quality of life" (very good or good) versus "poor quality of life" (not good nor bad, bad, or very bad).

Feelings of loneliness were assessed in both the full and short interviews by asking 
"Do you feel yourself lonely?" with answer options "never", "rarely", "sometimes", "often", and "constantly", dichotomised into "feelings of loneliness" (often or constantly) versus "no feelings of loneliness" (never, rarely, or sometimes).

\section{Outcome variables}

In the present study the outcome variable of integration was operationalized by six variables: current employment status, Finnish or Swedish language skills, voting, Finnish media use, having Finnish friends, and participation in leisure activities. In the bidirectional process of integration, the present study included only the minor groups' perspectives.

Current employment status was assessed in both the full and short interviews by asking "Which of the following options describes the best your main activity at the moment" with answer options "full-time job", "part-time job", "student", "retired", "unemployed", "home-stay parent", and "other", dichotomised into "employed or student" (full-time job, part-time job, or student) versus "unemployed or economically inactive" (retired, unemployed, home-stay parent, or other).

Language skills were assessed in both the full and short interviews by asking "Do you understand spoken Finnish/Swedish language?" with answer options "not at all", "poorly", "moderate", and "well", dichotomised into "good command in Finnish/Swedish language" (well or moderate) and "poor command in Finnish/Swedish language" (not at all or poorly).

Voting was assessed in the full interview by asking "Did you vote in the last parliament elections?" from those with Finnish citizenship and thus eligible to vote, with answer options "yes" and "no".

Finnish media use was assessed in the full interview by asking "How often do you follow the happenings of Finland from newspapers, radio, Internet, or television?" with answer options "daily", "weekly", "monthly", or "rarely", dichotomised into "following Finnish media" (daily) versus "not following Finnish media" (weekly, monthly, or rarely).

The number of Finnish friends was assessed in the full interview by asking "How many good friends you have?" with a follow-up question "How many of these friends are of Finns origin?", combined and dichotomised into "having good Finnish friends" (one or more) versus "not having good Finnish friends" (none).

Participation in leisure activities were assessed in the interview by asking "How many times have you participated in the activities of the following organizations, associations, societies, or others during the last 12 months?" with seven sub-categories such as sport clubs, culture associations, religious communities, and informal hobby groups, each answered with a 5-point scale ranging from "three times per week or more" to "not taken part in any activity". The questions were combined and dichotomized into "active participation in leisure activities" (at least one of the activities at least once per month) versus "not active participation in leisure activities" (none of the activities at least once per month). 


\section{Statistical analyses}

The statistical analyses were performed using SAS 9.3 and SUDAAN 11.0.1 programs. Inverse probablility weights (IPW) were calculated for the sample using register information for age, gender, population group, municipality, and marital status (Castaneda et al. 2012). The weights were used in all analyses in order to produce estimates that are representative to the Russian, Somali, and Kurdish origin groups. The finite population correction was used since a significant proportion of the total population was included in the sample (Lehtonen and Pahkinen 2004).

Logistic regression analyses were conducted to examine the associations between pre- and post-migration wellbeing factors (predictors) and integration outcomes. All of the predictors were examined separately with each outcome variable. All logistic regression analyses were adjusted for age. The results are presented as odds ratios (OR) with $95 \%$ confidence interval (CI). P-value of $<0.05$ was considered statistically significant.

\section{Ethical considerations}

The Maamu Study has been approved by the Coordinating Ethical Committee of the Helsinki and Uusimaa Hospital District, Finland. Written informed consent was collected from each participant.

\section{Results}

The distributions of all the variables used in the present study are presented in Table 1. The associations between the pre- and post-migration wellbeing factors and integration outcomes are presented in Tables 2, 3 and 4 for Russian, Somali, and Kurdish origin participants, respectively.

\section{Associations between basic education and integration outcomes}

There were no associations between basic educational level and integration outcomes among the Russian men or women.

Among the Somali origin men, the basic educational level associated with voting and active participation in leisure activities. Among Somali origin women, the basic educational level associated with all of the explored integration outcomes other than voting. Those with high basic education were more often employed or studying, voted more often, followed Finnish media more often, had more often Finnish friends, and were more likely to actively participate in leisure activities.

Among Kurdish origin participants, basic education associated with Finnish or Swedish language skills in both men and women. In addition, basic education associated with employment status in Kurdish origin men, and having Finnish friends and participating in leisure activities in Kurdish origin women. Those with high school education were more often employed or studying, had more often good command on Finnish or Swedish, had more often Finnish friends, and were more likely to actively participate in leisure activities. 
Table 1. Variable distributions in the study samples.

\begin{tabular}{|c|c|c|c|c|c|c|}
\hline & $\mathbf{N}$ & $\begin{array}{l}\text { Russian } \\
\%(95 \% \mathrm{Cl})\end{array}$ & $\mathbf{N}$ & $\begin{array}{l}\text { Somali } \\
\%(95 \% \mathrm{Cl})\end{array}$ & $\mathbf{N}$ & $\begin{array}{l}\text { Kurdish } \\
\%(95 \% \mathrm{Cl})\end{array}$ \\
\hline \multicolumn{7}{|l|}{ MEN } \\
\hline Age: 18-29 & 253 & $34.0(27.8-40.8)$ & 218 & $42.1(35.0-49.6)$ & 326 & $40.3(35.5-45.4)$ \\
\hline Age: $30-39$ & & $33.5(27.4-40.1)$ & & 38.8 (31.9-46.3) & & $39.9(35.1-44.8)$ \\
\hline Age: $40-64$ & & $32.5(26.6-39.1)$ & & $19.0(14.0-25.2)$ & & $19.8(16.3-23.9)$ \\
\hline \multicolumn{7}{|l|}{ Basic education: } \\
\hline High school & 246 & $70.0(63.3-75.9)$ & 206 & $41.3(34.0-48.9)$ & 323 & $43.7(38.7-48.7)$ \\
\hline $\begin{array}{l}\text { Potentially traumatic } \\
\text { experiences: No }\end{array}$ & 188 & $73.6(66.3-79.8)$ & 153 & $58.6(49.7-67.0)$ & 278 & $19.2(15.1-24.1)$ \\
\hline Quality of life: Good & 188 & $82.4(75.6-87.6)$ & 151 & 97.5 (93.5-99.1) & 277 & $74.2(69.2-78.7)$ \\
\hline Feelings of loneliness: No & 249 & 95.5 (91.5-97.6) & 213 & $94.1(90.2-96.5)$ & 326 & $80.2(75.9-83.9)$ \\
\hline $\begin{array}{l}\text { Employment status: } \\
\text { Employed or student }\end{array}$ & 252 & $74.5(68.1-80.0)$ & 207 & $69.5(62.3-76.0)$ & 325 & $67.7(62.9-72.1)$ \\
\hline $\begin{array}{l}\text { Command on Finnish/ } \\
\text { Swedish: Good }\end{array}$ & 251 & $86.3(81.2-90.3)$ & 212 & $93.8(90.4-96.0)$ & 324 & $88.0(84.6-90.7)$ \\
\hline Voted in last elections: Yes & 73 & $44.1(31.9-57.1)$ & 51 & $71.1(56.5-82.3)$ & 103 & $47.0(38.2-55.9)$ \\
\hline Following Finnish media: Yes & 188 & $62.0(53.9-69.5)$ & 151 & $90.3(86.2-93.3)$ & 278 & $70.0(64.7-74.8)$ \\
\hline Finnish friends: At least one & 186 & $35.9(28.4-44.0)$ & 149 & $63.8(55.1-71.7)$ & 273 & $24.2(19.8-29.4)$ \\
\hline $\begin{array}{l}\text { Active participation in } \\
\text { leisure activities: Yes }\end{array}$ & 188 & $55.1(47.1-62.9)$ & 149 & $81.3(74.6-86.6)$ & 276 & $31.3(26.5-36.7)$ \\
\hline \multicolumn{7}{|l|}{ WOMEN } \\
\hline Age: 18-29 & 439 & $23.7(19.6-28.3)$ & 271 & $38.4(32.5-44.8)$ & 288 & $32.1(27.4-37.2)$ \\
\hline Age: $30-39$ & & $33.6(29.0-38.5)$ & & 39.7 (33.7-45.9) & & $45.0(39.8-50.2)$ \\
\hline Age: 40-64 & & $42.8(37.9-47.8)$ & & $21.9(17.3-27.3)$ & & $22.9(18.8-27.6)$ \\
\hline Basic education: High school & 428 & $81.0(76.6-84.7)$ & 260 & $16.6(12.3-22.0)$ & 283 & $41.9(36.8-47.2)$ \\
\hline $\begin{array}{l}\text { Potentially traumatic } \\
\text { experiences: No }\end{array}$ & 341 & $77.9(72.6-82.4)$ & 184 & $32.8(26.1-40.3)$ & 229 & $28.8(23.7-34.6)$ \\
\hline Quality of life: Good & 340 & $83.9(79.2-87.7)$ & 182 & $94.8(90.5-97.2)$ & 230 & $76.6(71.2-81.2)$ \\
\hline Feelings of loneliness: No & 437 & $93.6(90.5-95.7)$ & 259 & $93.3(89.9-95.6)$ & 288 & $76.0(71.1-80.2)$ \\
\hline $\begin{array}{l}\text { Employment status: } \\
\text { Employed or student }\end{array}$ & 438 & $65.7(60.7-70.3)$ & 261 & $39.4(33.3-45.8)$ & 286 & $54.3(49.1-59.5)$ \\
\hline $\begin{array}{l}\text { Command on Finnish/ } \\
\text { Swedish: Good }\end{array}$ & 438 & $91.6(88.4-93.9)$ & 266 & $72.3(66.3-77.5)$ & 287 & $80.1(75.6-83.9)$ \\
\hline Voted in last elections: Yes & 165 & $43.2(35.2-51.5)$ & 65 & $32.2(21.9-44.5)$ & 124 & $44.0(36.2-52.3)$ \\
\hline Following Finnish media: Yes & 341 & $68.0(62.4-73.2)$ & 179 & $65.0(57.4-71.9)$ & 230 & $59.7(53.7-65.4)$ \\
\hline Finnish friends: At least one & 338 & $36.8(31.3-42.7)$ & 166 & $24.3(17.9-32.1)$ & 230 & $35.3(29.8-41.1)$ \\
\hline $\begin{array}{l}\text { Active participation in } \\
\text { leisure activities: Yes }\end{array}$ & 341 & $50.8(45.0-56.5)$ & 180 & $51.4(43.8-59.0)$ & 230 & $26.2(21.2-31.8)$ \\
\hline
\end{tabular}




\section{Associations between potentially traumatic experiences and integration outcomes}

An association between potentially traumatic experiences and integration outcomes was observed in Russian origin women, with those not having potentially traumatic experiences in their former home country being more likely to follow Finnish media. Another association between potentially traumatic experiences and integration outcomes was observed in Somali origin men, with those not having potentially traumatic experiences in their former home country being more likely to have good command on Finnish or Swedish. No other statistically significant associations were observed.

Among Russian origin men, Somali origin women, and Kurdish origin men and women, potentially traumatic experiences did not associate with any of the integration outcomes.

\section{Associations between quality of life and integration outcomes}

Among Russian origin men and women good quality of life associated more often with being employed or studying. Among Russian origin women good quality of life associated also with following Finnish media more often.

Among Kurdish origin men good quality of life associated with more often being employed or studying and having good command on Finnish or Swedish. Among Kurdish origin women, those with good quality of life were more likely to be employed or studying and had voted more often.

Among Somali origin participants, the analyses of quality of life could not be performed due to too few observations.

\section{Associations between feelings of loneliness and integration outcomes}

Having less feelings of loneliness associated with having more often good command on Finnish or Swedish among Russian origin men and with following more often Finnish media among Russian women.

Among Somali origin men, having less feelings of loneliness associated with all of the explored integration outcomes other than employment status. Those with less feelings of loneliness had more often good command on Finnish or Swedish, followed Finnish media more often, had more often Finnish friends, and were more likely to actively participate in leisure activities. Among Somali origin women, having less feelings of loneliness associated with following more often Finnish media and having more often Finnish friends.

Among Kurdish origin women, having less feelings of loneliness associated with being more often employed or studying, voting, following Finnish media, and having Finnish friends. No associations were observed between feelings of loneliness and integration outcomes among Kurdish origin men. 
Table 2. Associations between pre- and post-migration wellbeing factors and integration outcomes for Russian origin men and women.

\begin{tabular}{|c|c|c|c|c|c|c|}
\hline & $\begin{array}{l}\text { Employed } \\
\text { or } \\
\text { student } \\
\text { OR }(95 \% \mathrm{Cl})\end{array}$ & $\begin{array}{l}\text { Good command } \\
\text { on Finnish/ } \\
\text { Swedish } \\
\text { OR }(95 \% \mathrm{Cl})\end{array}$ & $\begin{array}{l}\text { Voted } \\
\text { in last } \\
\text { elections } \\
\text { OR }(95 \% \mathrm{Cl})\end{array}$ & $\begin{array}{l}\text { Following } \\
\text { Finnish } \\
\text { media } \\
\text { OR }(95 \% \mathrm{Cl})\end{array}$ & $\begin{array}{l}\text { Having } \\
\text { Finnish } \\
\text { friends } \\
\text { OR }(95 \% \mathrm{Cl})\end{array}$ & $\begin{array}{l}\text { Active } \\
\text { participation in } \\
\text { leisure activities } \\
\text { OR }(95 \% \mathrm{Cl})\end{array}$ \\
\hline \multicolumn{7}{|l|}{ MEN } \\
\hline \multicolumn{7}{|l|}{$\begin{array}{l}\text { Pre-migration } \\
\text { wellbeing factors }\end{array}$} \\
\hline $\begin{array}{l}\text { Basic education } \\
\text { Comprehensive } \\
\text { school or lower } \\
\text { High school }\end{array}$ & $\begin{array}{l}1.00 \\
1.75(0.86-3.55)\end{array}$ & $\begin{array}{l}1.00 \\
1.43(0.62-3.27)\end{array}$ & $\begin{array}{l}1.00 \\
1.82(0.53-6.30)\end{array}$ & $\begin{array}{l}1.00 \\
0.94(0.43-2.06)\end{array}$ & $\begin{array}{l}1.00 \\
0.58(0.25-1.33)\end{array}$ & $\begin{array}{l}1.00 \\
1.94(0.87-4.33)\end{array}$ \\
\hline $\begin{array}{l}\text { Potentially } \\
\text { traumatic } \\
\text { experiences } \\
\text { Yes } \\
\text { No }\end{array}$ & $\begin{array}{l}1.00 \\
0.76(0.32-1.82)\end{array}$ & $\begin{array}{l}1.00 \\
0.69(0.24-2.00)\end{array}$ & $\begin{array}{l}1.00 \\
0.93(0.28-3.12)\end{array}$ & $\begin{array}{l}1.00 \\
0.89(0.43-1.83)\end{array}$ & $\begin{array}{l}1.00 \\
0.70(0.33-1.50)\end{array}$ & $\begin{array}{l}1.00 \\
1.42(0.70-2.86)\end{array}$ \\
\hline $\begin{array}{l}\text { Post-migration } \\
\text { wellbeing factors }\end{array}$ & & & & & & \\
\hline $\begin{array}{l}\text { Quality of life } \\
\text { Poor } \\
\text { Good }\end{array}$ & $\begin{array}{l}1.00 \\
3.48(1.41-8.58)\end{array}$ & $\begin{array}{l}1.00 \\
1.09(0.36-3.31) \\
\end{array}$ & $\begin{array}{l}1.00 \\
0.88(0.23-3.38)\end{array}$ & $\begin{array}{l}1.00 \\
0.88(0.36-2.18) \\
\end{array}$ & $\begin{array}{l}1.00 \\
0.73(0.28-1.94) \\
\end{array}$ & $\begin{array}{l}1.00 \\
1.21(0.51-2.85) \\
\end{array}$ \\
\hline $\begin{array}{l}\text { Feelings of } \\
\text { loneliness } \\
\text { Yes } \\
\text { No }\end{array}$ & $\begin{array}{l}1.00 \\
2.43(0.82-7.21)\end{array}$ & $\begin{array}{l}1.00 \\
4.59(1.04-20.20)\end{array}$ & NA & NA & NA & NA \\
\hline WOMEN & & & & & & \\
\hline $\begin{array}{l}\text { Pre-migration } \\
\text { wellbeing factors }\end{array}$ & & & & & & \\
\hline $\begin{array}{l}\text { Basic education } \\
\text { Comprehensive } \\
\text { school or lower } \\
\text { High school }\end{array}$ & $\begin{array}{l}1.00 \\
1.10(0.63-1.91)\end{array}$ & $\begin{array}{l}1.00 \\
1.36(0.57-3.24)\end{array}$ & $\begin{array}{l}1.00 \\
1.08(0.43-2.70)\end{array}$ & $\begin{array}{l}1.00 \\
1.18(0.58-2.42)\end{array}$ & $\begin{array}{l}1.00 \\
0.80(0.41-1.56)\end{array}$ & $\begin{array}{l}1.00 \\
1.10(0.60-2.05)\end{array}$ \\
\hline $\begin{array}{l}\text { Potentially } \\
\text { traumatic } \\
\text { experiences } \\
\text { Yes } \\
\text { No }\end{array}$ & $\begin{array}{l}1.00 \\
1.47(0.81-2.68)\end{array}$ & $\begin{array}{l}1.00 \\
1.90(0.79-4.60)\end{array}$ & $\begin{array}{l}1.00 \\
0.96(0.45-2.08)\end{array}$ & $\begin{array}{l}1.00 \\
2.00(1.07-3.75)\end{array}$ & $\begin{array}{l}1.00 \\
0.64(0.35-1.17)\end{array}$ & $\begin{array}{l}1.00 \\
0.56(0.31-1.00)\end{array}$ \\
\hline $\begin{array}{l}\text { Post-migration } \\
\text { wellbeing factors }\end{array}$ & & & & & & \\
\hline $\begin{array}{l}\text { Quality of life } \\
\text { Poor } \\
\text { Good }\end{array}$ & $\begin{array}{l}1.00 \\
2.76(1.44-5.27)\end{array}$ & $\begin{array}{l}1.00 \\
2.16(0.93-5.02) \\
\end{array}$ & $\begin{array}{l}1.00 \\
1.51(0.58-3.93)\end{array}$ & $\begin{array}{l}1.00 \\
2.27(1.14-4.52)\end{array}$ & $\begin{array}{l}1.00 \\
1.93(0.98-3.82) \\
\end{array}$ & $\begin{array}{l}1.00 \\
1.71(0.90-3.25)\end{array}$ \\
\hline $\begin{array}{l}\text { Feelings of } \\
\text { loneliness } \\
\text { Yes } \\
\text { No }\end{array}$ & $\begin{array}{l}1.00 \\
1.06(0.44-2.55)\end{array}$ & $\begin{array}{l}1.00 \\
1.82(0.56-5.91)\end{array}$ & $\begin{array}{l}1.00 \\
2.06(0.64-6.56)\end{array}$ & $\begin{array}{l}1.00 \\
4.65(1.81-11.95)\end{array}$ & $\begin{array}{l}1.00 \\
3.24(0.97-10.81)\end{array}$ & $\begin{array}{l}1.00 \\
1.22(0.46-3.22)\end{array}$ \\
\hline
\end{tabular}

Age-adjusted analysis. NA=Too few observations to perform the analysis. 
Table 3. Associations between pre-and post-migration wellbeing factors and integration outcomes for Somali origin men and women.

\begin{tabular}{|c|c|c|c|c|c|c|}
\hline & $\begin{array}{l}\text { Employed } \\
\text { or } \\
\text { student } \\
\text { OR }(95 \% \mathrm{Cl})\end{array}$ & $\begin{array}{l}\text { Good command } \\
\text { on Finnish/ } \\
\text { Swedish } \\
\text { OR }(95 \% \mathrm{Cl})\end{array}$ & $\begin{array}{l}\text { Voted } \\
\text { in last } \\
\text { elections } \\
\text { OR }(95 \% \mathrm{Cl})\end{array}$ & $\begin{array}{l}\text { Following } \\
\text { Finnish } \\
\text { media } \\
\text { OR (95\% Cl) }\end{array}$ & $\begin{array}{l}\text { Having } \\
\text { Finnish } \\
\text { friends } \\
\text { OR }(95 \% \mathrm{Cl})\end{array}$ & $\begin{array}{l}\text { Active } \\
\text { participation in } \\
\text { leisure activities } \\
\text { OR }(95 \% \mathrm{Cl})\end{array}$ \\
\hline \multicolumn{7}{|l|}{ MEN } \\
\hline \multicolumn{7}{|l|}{$\begin{array}{l}\text { Pre-migration } \\
\text { wellbeing factors }\end{array}$} \\
\hline $\begin{array}{l}\text { Basic education } \\
\text { Comprehensive } \\
\text { school or lower } \\
\text { High school }\end{array}$ & $\begin{array}{l}1.00 \\
1.42(0.64-3.16)\end{array}$ & NA & $\begin{array}{l}1.00 \\
8.42(1.99-35.67)\end{array}$ & NA & $\begin{array}{l}1.00 \\
0.93(0.43-2.02)\end{array}$ & \begin{tabular}{|l}
1.00 \\
$6.47(2.06-20.39)$
\end{tabular} \\
\hline \multicolumn{7}{|l|}{$\begin{array}{l}\text { Potentially } \\
\text { traumatic } \\
\text { experiences }\end{array}$} \\
\hline \multicolumn{7}{|l|}{$\begin{array}{l}\text { Post-migration } \\
\text { wellbeing factors }\end{array}$} \\
\hline $\begin{array}{l}\text { Quality of life } \\
\text { Poor } \\
\text { Good }\end{array}$ & NA & NA & NA & NA & NA & NA \\
\hline $\begin{array}{l}\text { Feelings } \\
\text { of loneliness } \\
\text { Yes } \\
\text { No }\end{array}$ & $\begin{array}{l}1.00 \\
1.01(0.25-4.02)\end{array}$ & $\begin{array}{l}1.00 \\
7.30(1.77-30.08)\end{array}$ & $\begin{array}{l}\text { NA } \\
5.28(1.60-17.42)\end{array}$ & $\begin{array}{l}1.00 \\
8.51(1.66-43.64)\end{array}$ & $\begin{array}{l}1.00 \\
5.01(1.46-17.21)\end{array}$ & 1.00 \\
\hline \multicolumn{7}{|l|}{ WOMEN } \\
\hline \multicolumn{7}{|l|}{$\begin{array}{l}\text { Pre-migration } \\
\text { wellbeing factors }\end{array}$} \\
\hline $\begin{array}{l}\text { Basic education } \\
\text { Comprehensive } \\
\text { school or lower } \\
\text { High school }\end{array}$ & $\begin{array}{l}1.00 \\
2.41(1.17-4.95)\end{array}$ & $\begin{array}{l}\text { NA } \\
2.64(0.80-8.74)\end{array}$ & $\begin{array}{l}1.00 \\
3.65(1.01-13.16)\end{array}$ & $\begin{array}{l}1.00 \\
3.45(1.34-8.90)\end{array}$ & $\begin{array}{l}1.00 \\
2.64(1.05-6.66)\end{array}$ & 1.00 \\
\hline $\begin{array}{l}\text { Potentially } \\
\text { traumatic } \\
\text { experiences } \\
\text { Yes } \\
\text { No }\end{array}$ & $\begin{array}{l}1.00 \\
0.55(0.25-1.20)\end{array}$ & $\begin{array}{l}1.00 \\
1.01(0.44-2.29)\end{array}$ & $\begin{array}{l}1.00 \\
0.83(0.25-2.79)\end{array}$ & $\begin{array}{l}1.00 \\
1.28(0.64-2.56)\end{array}$ & $\begin{array}{l}1.00 \\
2.17(0.94-5.02)\end{array}$ & \begin{tabular}{|l}
1.00 \\
$1.02(0.50-2.05)$
\end{tabular} \\
\hline $\begin{array}{l}\text { Post-migration } \\
\text { wellbeing factors }\end{array}$ & & & & & & \\
\hline $\begin{array}{l}\text { Quality of life } \\
\text { Poor } \\
\text { Good }\end{array}$ & NA & NA & NA & NA & NA & NA \\
\hline $\begin{array}{l}\text { Feelings } \\
\text { of loneliness } \\
\text { Yes } \\
\text { No }\end{array}$ & $\begin{array}{l}1.00 \\
0.51(0.21-1.24)\end{array}$ & $\begin{array}{l}1.00 \\
1.74(0.69-4.41)\end{array}$ & NA & $\begin{array}{l}1.00 \\
7.50(2.12-26.60)\end{array}$ & $\begin{array}{l}1.00 \\
3.57(1.08-11.78)\end{array}$ & NA \\
\hline
\end{tabular}

Age-adjusted analysis.

$\mathrm{NA}=$ Too few observations to perform the analysis. 
Table 4. Associations between pre- and post-migration wellbeing factors and integration outcomes for Kurdish origin men and women.

\begin{tabular}{|c|c|c|c|c|c|c|}
\hline & $\begin{array}{l}\text { Employed } \\
\text { or } \\
\text { student } \\
\text { OR }(95 \% \mathrm{Cl})\end{array}$ & $\begin{array}{l}\text { Good command } \\
\text { on Finnish/ } \\
\text { Swedish } \\
\text { OR }(95 \% \mathrm{Cl})\end{array}$ & $\begin{array}{l}\text { Voted } \\
\text { in last } \\
\text { elections } \\
\text { OR }(95 \% \mathrm{Cl})\end{array}$ & $\begin{array}{l}\text { Following } \\
\text { Finnish } \\
\text { media } \\
\text { OR (95\% Cl) }\end{array}$ & $\begin{array}{l}\text { Having } \\
\text { Finnish } \\
\text { friends } \\
\text { OR }(95 \% \mathrm{Cl})\end{array}$ & $\begin{array}{l}\text { Active } \\
\text { participation in } \\
\text { leisure activities } \\
\text { OR }(95 \% \mathrm{Cl})\end{array}$ \\
\hline \multicolumn{7}{|l|}{ MEN } \\
\hline \multicolumn{7}{|l|}{$\begin{array}{l}\text { Pre-migration } \\
\text { wellbeing factors }\end{array}$} \\
\hline \multicolumn{7}{|l|}{ Basic education } \\
\hline $\begin{array}{l}\text { Comprehensive } \\
\text { school or lower } \\
\text { High school }\end{array}$ & $\begin{array}{l}1.00 \\
1.94(1.25-3.02)\end{array}$ & $\begin{array}{l}1.00 \\
2.84(1.44-5.62)\end{array}$ & $\begin{array}{l}1.00 \\
1.47(0.67-3.25)\end{array}$ & $\begin{array}{l}1.00 \\
1.10(0.66-1.83)\end{array}$ & $\begin{array}{l}1.00 \\
0.78(0.46-1.33)\end{array}$ & $\begin{array}{l}1.00 \\
1.57(0.95-2.59)\end{array}$ \\
\hline \multicolumn{7}{|l|}{$\begin{array}{l}\text { Potentially } \\
\text { traumatic } \\
\text { experiences }\end{array}$} \\
\hline Yes & 1.00 & 1.00 & 1.00 & 1.00 & 1.00 & 1.00 \\
\hline No & $1.07(0.55-2.07)$ & $1.79(0.52-6.13)$ & $0.85(0.26-2.77)$ & $1.19(0.62-2.29)$ & $1.22(0.60-2.49)$ & $1.18(0.62-2.25)$ \\
\hline \multicolumn{7}{|l|}{$\begin{array}{l}\text { Post-migration } \\
\text { wellbeing factors }\end{array}$} \\
\hline \multicolumn{7}{|l|}{ Quality of life } \\
\hline Poor & 1.00 & 1.00 & 1.00 & 1.00 & 1.00 & 1.00 \\
\hline Good & $2.21(1.29-3.79)$ & $2.28(1.04-5.01)$ & $1.38(0.53-3.58)$ & $1.19(0.67-2.10)$ & $1.80(0.99-3.28)$ & $1.22(0.70-2.11)$ \\
\hline \multicolumn{7}{|l|}{$\begin{array}{l}\text { Feelings } \\
\text { of loneliness }\end{array}$} \\
\hline Yes & 1.00 & 1.00 & 1.00 & 1.00 & 1.00 & 1.00 \\
\hline No & $0.78(0.43-1.43)$ & $1.70(0.82-3.51)$ & $1.10(0.36-3.33)$ & $1.27(0.70-2.30)$ & $2.00(0.90-4.45)$ & $1.29(0.70-2.37)$ \\
\hline \multicolumn{7}{|l|}{ WOMEN } \\
\hline \multicolumn{7}{|l|}{$\begin{array}{l}\text { Pre-migration } \\
\text { wellbeing factors }\end{array}$} \\
\hline \multicolumn{7}{|l|}{ Basic education } \\
\hline $\begin{array}{l}\text { Comprehensive } \\
\text { school or lower }\end{array}$ & 1.00 & 1.00 & 1.00 & 1.00 & 1.00 & 1.00 \\
\hline High school & $1.13(0.73-1.75)$ & $2.66(1.44-4.91)$ & $1.13(0.57-2.21)$ & $1.45(0.88-2.38)$ & $3.04(1.80-5.13)$ & $2.82(1.59-5.01)$ \\
\hline \multicolumn{7}{|l|}{$\begin{array}{l}\text { Potentially } \\
\text { traumatic } \\
\text { experiences }\end{array}$} \\
\hline Yes & 1.00 & 1.00 & 1.00 & 1.00 & 1.00 & 1.00 \\
\hline No & $1.03(0.54-1.95)$ & $2.15(0.86-5.37)$ & $1.09(0.44-2.72)$ & $1.84(0.95-3.55)$ & $1.13(0.61-2.11)$ & $0.89(0.43-1.86)$ \\
\hline \multicolumn{7}{|l|}{$\begin{array}{l}\text { Post-migration } \\
\text { wellbeing factors }\end{array}$} \\
\hline \multicolumn{7}{|l|}{ Quality of life } \\
\hline Poor & 1.00 & 1.00 & 1.00 & 1.00 & 1.00 & 1.00 \\
\hline Good & $3.21(1.73-5.96)$ & $1.52(0.75-3.10)$ & $1.76(0.83-3.73)$ & $1.83(1.02-3.27)$ & $1.12(0.60-2.09)$ & $1.34(0.67-2.66)$ \\
\hline \multicolumn{7}{|l|}{$\begin{array}{l}\text { Feelings } \\
\text { of loneliness }\end{array}$} \\
\hline Yes & 1.00 & 1.00 & 1.00 & 1.00 & 1.00 & 1.00 \\
\hline No & $1.89(1.12-3.19)$ & $1.66(0.90-3.06)$ & $2.99(1.17-7.63)$ & $2.40(1.33-4.33)$ & $2.40(1.21-4.76)$ & $1.57(0.77-3.19)$ \\
\hline
\end{tabular}

Age-adjusted analysis. 


\section{Discussion}

The aim of this study was to investigate the associations between pre- and post-migration wellbeing factors with integration outcomes among Russian, Somali, and Kurdish origin men and women in Finland. As hypothesized, protective pre- and post-migration situations and social wellbeing factors were associated with better integration outcomes: integration outcomes were associated with feelings of loneliness and quality of life in Russian origin men; potentially traumatic experiences, quality of life, and loneliness in Russian origin women; basic education, potentially traumatic experiences, and loneliness in Somali origin men; basic education and loneliness in Somali origin women; basic education and quality of life in Kurdish origin men; and basic education, quality of life, and loneliness in Kurdish origin women.

Among Kurdish origin men and women and especially among Somali origin men and women, basic education explained many of the integration outcomes. This was an expected finding, since education gained in the former home country has been seen as a promotor for social integration in a new home country in previous studies, too (Martinovic, Tubergen and van Maas 2009). Educated persons have been thought to have more intellectual recourses to cope with problems and challenges in the new host society (Lueck and Wilson 2010). Regarding the present study, it should be noted that some of the basic educations might be completed in Finland, too, with those who have migrated in early stages of life, and therefore this is not entirely pre-migration aspect only. This should be investigated more. All the activities that promote additional education for migrant origin men and women with lower basic education are important to promote overall integration. The same result was not evident among the Russian origin men nor women, which might be explained by the high number of those having high school education. It might be that the operationalization used in the present study was not the most applicable for the Russian origin participants with very different educational background that the Somali or Kurdish origin participants.

Even though Somali and Kurdish origin men and women have high levels of potentially traumatic experiences in their former home countries (Castaneda et al. 2017), the results of the present study did not show many associations between potentially traumatic experiences and integration outcomes in the two groups. The only associations observed were having no potentially traumatic experiences associating with following Finnish media more often among Russian origin women and having good command in Finnish or Swedish among Somali origin men. Although these single findings are important, the lack of stronger associations was somewhat surprising, and might be explained with the high level of those having different types and severities of potentially traumatic experiences in their former home country, from milder single experiences to cumulative and more complex traumatization. Future research should investigate more these aspects. However, it is good news that the role of the difficult experiences in the individual's past might not have such a definitive role in integration to the new society.

Good quality of life associated with being employed or studying among Russian and Kurdish origin men and women, following Finnish media among the Russian and Kurdish origin women, and having good command in Finnish of Swedish among Kurd- 
ish origin men. It is important to notice that although quality of life is a factor reflecting post-migration life in the new host country and in the present study's analyses defined as an explanatory variable, it may as well be seen as an outcome of integration process. All in all, it is important to notice the strong associations between quality of life and integration among the Russian and Kurdish origin people.

Loneliness was associated with several integration outcomes in all groups investigated other than Kurdish origin men. Again, this association might in real life operate in both ways, and the important finding stresses the need for reducing loneliness to promote integration and vice versa. It may also be difficult to clearly distinguish the two phenomena of social wellbeing and integration, since both have some similar components. For example loneliness as a predictor in the present study is, regarding the contents, quite close to some of the outcomes, too, such as having Finnish friends or participating actively in leisure activities. Thus it is not surprising that the two are interconnected, but still stresses the importance to support all of these aspects.

\section{Strengths and limitations}

A clear strength of this study is the population-based study design and the possibility to conduct the analyses separately for the three study groups and genders that vary in their characteristics. Another strength is a relatively high participation rate compared to other surveys conducted among migrant origin groups, although non-response may still cause some bias in the results, particularly among the Somali origin participants for whom the participation rate was the lowest.

Furthermore, the study design is cross-sectional, and therefore it is not possible to draw conclusions about any causalities. For example, good quality of life may account in being employed and vice versa. Another limitation is that some of the used scales and questions have not been evaluated in cross-cultural settings. Therefore, it may vary whether for example the concept of quality of life or the definition of a "good friend" is being understood similarly in the three groups.

\section{Conclusion}

The results of the present study revealed that integration outcomes were associated with only post-migration factors in Russian origin men, potentially traumatic experiences and post-migration factors in Russian origin women, pre-migration factors and loneliness in Somali origin men, basic education and loneliness in Somali origin women, basic education and quality of life in Kurdish origin men, and basic education, quality of life, and loneliness in Kurdish origin women. Thus, both pre- and post-migration factors are important to be taken into account in integration discourse. Future studies should go into deeper to quantitatively investigate the more fine-tuned aspects of for example traumatization or basic education in integration outcomes, to widen the aspects of post-migration factors to for example sense of belongingness, to qualitatively understand more of the phenomena, and to explore more of the major groups' role in integration processes. 


\section{References}

Berry, J. W. (1997). Immigration, acculturation, and adaptation. Applied Psychology $46(1), 5-34$.

Berry, J. W. (2005). Acculturation: living successfully in two cultures. International Journal of Intercultural Relations 29(6), 697-712.

Castaneda, A. E., Rask, S., Koponen, P., Mölsä, M., \& Koskinen, S. (editors) (2012). Maahanmuuttajien terveys ja hyvinvointi. Tutkimus venäläis-, somalialais-ja kurditaustaisista Suomessa [Migrant health and wellbeing. A study on persons of Russian, Somali and Kurdish origin in Finland]. Raportti, 61. Available from: http:// www.julkari.fi/handle/10024/90907

Castaneda, A. E., Rask, S., Koponen, P., Suvisaari, J., Koskinen, S., Härkänen, T., Mannila, S., Laitinen, K., Jukarainen, P., \& Jasinskaja-Lahti, I. (2015). The Association between discrimination and psychological and social well-being: a population-based study of Russian, Somali and Kurdish migrants in Finland. Psychology \& Developing Societies 27(2), 270-92.

Castaneda, A. E., Junna, L., Lilja, E., Skogberg, N., Kuusio, H., Koponen, P., \& Suvisaari, J. (2017). The prevalence of potentially traumatic pre-migration experiences in Russian, Somali and Kurdish origin migrants in Finland: a population-based study in Finland. Journal of Traumatic Stress Disorders and Treatment 6, 1.

Castaneda, A. E., Rask, S., Härkänen, T., Juntunen, T., Skogberg, N., Mölsä, M., Tolonen, H., Koskinen, S., \& Koponen, P. (2018). Enhancing survey participation among foreign-born populations. Experiences from the Finnish Migrant Health and Wellbeing Study (Maamu). Finnish Yearbook of Population Research 53, 89-110

Castaneda, A., \& Kuusio, H. (2019). Sosiaalinen hyvinvointi, kotoutuminen ja terveys sekä näiden väliset yhteydet Suomen ulkomailla syntyneessä väestössä [Social wellbeing, integration, health and the association between these in the foreign-born population in Finland]. In: Kazi, V., Alitolppa-Niitamo, A., Kaihovaara, A. (editors). Kotoutumisen kokonaiskatsaus 2019: Tutkimusartikkeleita kotoutumisesta. TEM oppaat ja muut julkaisut, 10. Helsinki: Työ- ja elinkeinoministeriö. http://urn.fi/ URN:ISBN:978-952-327-487-7

Lehtonen, R., \& Pahkinen, E. (2004). Practical methods for design and analysis of complex surveys. Revised, 2nd Edition. Chichester: John Wiley \& Sons.

Lueck, K., \& Wilson, M. (2010). Acculturative stress in Asian immigrants: the impact of social and linguistic factors. International Journal of Intercultural Relations 34(1), 47-57.

Martiniello, M. (2006). Towards a coherent approach to immigrant integration policy(ies) in the European Union. OECD Publications and Papers.

Martinovic, B., Tubergen, F., \& van Maas, I. (2009). Changes in immigrants' social integration during the stay in the host country: the case of non-western immigrants in the Netherlands. Social Science and Research 38(4), 870-82.

Ministry of Employment and the Economy (2010). Act on the Promotion of Immigrant Integration. 
Rask, S., Suvisaari, J., Koskinen, S., Koponen, P., Mölsä, M., Lehtisalo, R., Schubert, C., Pakaslahti, A., \& Castaneda, A. E. (2016a). The ethnic gap in mental health: a population-based study of Russian, Somali and Kurdish origin migrants in Finland. Scandinavian Journal of Public Health 44, 281-90.

Rask, S., Sainio, P., Castaneda, A. E., Koponen, P., \& Koskinen, S. (2016b). The ethnic gap in mobility: a population-based study of Russian, Somali and Kurdish origin migrants in Finland. BMC Public Health 16, 340.

Salama, E., Niemelä, S., Suvisaari, J., Laatikainen, T., Koponen, P., \& Castaneda, A. E. (2018). The prevalence of substance use among Russian, Somali and Kurdish migrants in Finland: a population-based study. BMC Public Health 18, 651.

Skeldon, R. (editor) (2013). Global migration: demographic aspects and its relevance for development. United Nations: Department of Economic and Social Affairs.

Skogberg, N., Adam, A., Kinnunen, T., Lilja, E., \& Castaneda, A. E. (2018). Overweight and obesity among Russian, Somali, and Kurdish origin populations in Finland. Finnish Yearbook of Population Research 53, 73-88.

Skogberg, N., \& Koponen, P. (2019). Terveyden yhteys työssäoloon koko väestössä ja ulkomaalaistaustaisilla [The association between health and employment in the general population and foreign-origin persons]. In: Kazi, V., Alitolppa-Niitamo, A., Kaihovaara, A. (editors). Kotoutumisen kokonaiskatsaus 2019: Tutkimusartikkeleita kotoutumisesta. TEM oppaat ja muut julkaisut, 10. Helsinki: Työ- ja elinkeinoministeriö. http://urn.fi/URN:ISBN:978-952-327-487-7

Statistics Finland (2020). Maahanmuuttajat ja kotoutuminen [Migrants and integration]. http://www.stat.fi/tup/maahanmuutto/index.html

United Nations (2016). International Migration Report 2015. Department of Economic and Social Affairs. 